\title{
Trends of Major Foodborne Outbreaks in the European Union during the Years 2015-2019
}

\author{
Maria Schirone * (D) and Pierina Visciano \\ Faculty of Bioscience and Technology for Food Agriculture and Environment, University of Teramo, \\ Via R. Balzarini 1, 64100 Teramo, Italy; pvisciano@unite.it \\ * Correspondence: mschirone@unite.it; Tel.: +39-0861-266911
}

Citation: Schirone, M.; Visciano, P. Trends of Major Foodborne Outbreaks in the European Union during the Years 2015-2019. Hygiene 2021, 1, 106-119. https://doi.org/ 10.3390/hygiene1030010

Academic Editor: Günter Kampf

Received: 27 September 2021

Accepted: 29 October 2021

Published: 2 November 2021

Publisher's Note: MDPI stays neutral with regard to jurisdictional claims in published maps and institutional affiliations.

Copyright: (c) 2021 by the authors. Licensee MDPI, Basel, Switzerland. This article is an open access article distributed under the terms and conditions of the Creative Commons Attribution (CC BY) license (https:// creativecommons.org/licenses/by/ $4.0 /)$.
Abstract: The incidence of the most common foodborne outbreaks reported by the European Food Safety Authority and the European Centre for Disease Prevention and Control during the years 2015-2019 is described. Campylobacter spp., Salmonella spp., Listeria monocytogenes, and Yersinia enterocolitica are the investigated microorganisms, and symptomatology, food categories responsible for human disease, as well as some prevention measures are the most important information schedules supplied to the readers. Campylobacteriosis and salmonellosis are the most common zoonoses with a notification rate of 59.7 and 20.0 per 100,000 population, respectively, in the year 2019. Good hygienic practices both at farm and domestic level could prevent such infections. The highest number of deaths is reported for listeriosis, corresponding to 31 fatal events in the year 2019. Therefore, awareness of the hazards linked to L. monocytogenes is particularly recommended amongst high-risk groups. By contrast, most cases of yersiniosis are sporadic and the most important prophylactic measures include adequate sanitation in pork chain, personnel hygiene, and protection of water supplies.

Keywords: food; outbreaks; Campylobacter spp.; Salmonella spp.; Listeria monocytogenes; Yersinia enterocolitica

\section{Introduction}

The foodborne diseases are caused by the consumption of food contaminated with microorganisms or their toxins, which may occur at any stage of the food supply chain, from production to distribution. They can also derive from the ingestion of chemicals, such as heavy metals or toxicants from plants (i.e., solanine, hydrogen cyanide, pyrrolizidine alkaloids, etc.), and animals (tetrodotoxin, scombrotoxin, and marine biotoxins). The microorganisms causing foodborne diseases can spread from person to person through the fecal-oral route, or arise from cross-contamination, and other factors associated with food production, such as poor personal hygiene, mixing of raw and cooked food, undercooking, and further conditions favoring microbial growth [1]. Even the raw material can be contaminated by air, soil, wildlife, irrigation water and manure [2]. The processing environments can be considered a serious source of contamination, especially due to the ineffective cleaning and disinfection procedures, and the environmental monitoring programs represent a valid strategy to improve food hygiene. By contrast, testing only the end-products might not be sufficient to guarantee their safety, as the negative result of microorganism presence does not mean its absence in the whole production [3].

The foodborne diseases can be distinguished in foodborne infections caused by microorganisms ingested through food and infecting the gastrointestinal tract by releasing toxins, damaging the intestinal epithelium, and causing gastroenteritis, and foodborne intoxications by ingesting food containing toxins produced by microorganisms, characterized by a short incubation period and lack of fever [1]. The pathogenic microorganisms can cause several health problems, such as repetitive intestinal infections, central nervous or renal disorders, arthritis, and blindness [4]. Some symptoms are characterized by hemorrhagic colitis and hemolytic uremic syndrome by Escherichia coli O157:H7, acute 
gastroenteritis by Campylobacter spp., Salmonella spp. and Vibrio parahaemolyticus, chronic arthritis by Shigella spp. and Salmonella spp., and abortion by Listeria monocytogenes. The World Health Organization reported around 600 million cases of foodborne diseases and 420,000 related deaths occurring annually due to pathogens [5].

Surveillance systems aiming at the monitoring of foodborne outbreaks occurring each year worldwide are imperative. Some examples are the Centers for Disease Control (CDC) and Prevention operating in the United States (US) since 1966, and the European Centre for Disease Prevention and Control (ECDC) for the Member States of the European Union (EU). A foodborne outbreak can be considered as a cluster of two or more diseases epidemiologically associated in time and/or space. The data collected through a surveillance system generally involve outbreak location, etiologic agent, patient demographics, number of cases and their severity, as well as the most likely foods associated to such events [6]. The aim of this review is the description of some of the most reported foodborne diseases caused by the following pathogens, i.e., Campylobacter spp., Salmonella spp., L. monocytogenes and Yersinia enterocolitica, their symptomatology and food sources.

\section{The EFSA-ECDC Annual Reports on Zoonoses and Foodborne Outbreaks}

The Zoonoses Directive 2003/99/EC requires that the EU Member States collect relevant data on zoonoses, zoonotic agents, antimicrobial resistance and foodborne outbreaks occurring each year in their territory. According to the EFSA-ECDC Annual Reports, the first and second most reported zoonoses in humans are campylobacteriosis and salmonellosis and their trend during 2015-2019 was stable. Specifically, campylobacteriosis is the most common zoonosis since 2005, representing 50\% of all the reported cases and corresponding to an EU notification rate of 59.7 per 100,000 population in the year 2019, whereas for salmonellosis such rate corresponds to 20.0 per 100,000 population. The third and fourth most reported zoonoses are respectively Shiga toxin-producing E. coli infection, and yersiniosis. By contrast, the most severe diseases defined in the EU Reports are listeriosis and the West Nile virus infection, with the highest case fatality and hospitalization, respectively.

The trend of confirmed human cases of the foodborne diseases selected in our review is quite stable for all of them (Figure 1). According to the EFSA technical specifications, the foodborne outbreaks can be distinguished in outbreaks supported by weak-evidence and those supported by strong-evidence, based on the strength of evidence implicating a particular food vehicle. Moreover, the nature of evidence linking the consumption of a particular food vehicle to an outbreak can be epidemiological, microbiological, descriptive environmental or based on product-tracing investigations. The strong microbiological evidence includes the identification of an indistinguishable causative agent in a human case and in a food vehicle, which is unlikely to have been contaminated coincidentally or after the event [7]. In Figure 2, the strong- and weak-evidence foodborne outbreaks per causative agent were shown. The number of deaths was not reported because it was high only for listeriosis, corresponding to two (in the years 2016 and 2017) and four in the year 2015, while 21 and 31 fatal events occurred in the year 2018 and 2019, respectively. For campylobacteriosis, only one death was reported in both years 2015 and 2017, whereas for salmonellosis the deaths ranged from one to 10 in the year 2016 [8].

The States notifying the investigated foodborne outbreaks are detailed in Table 1. Campylobacteriosis and salmonellosis were reported each year from almost all of them, while listeriosis and yersiniosis were rare events, and Sweden, Denmark, Finland, and Germany were the main advising countries. 


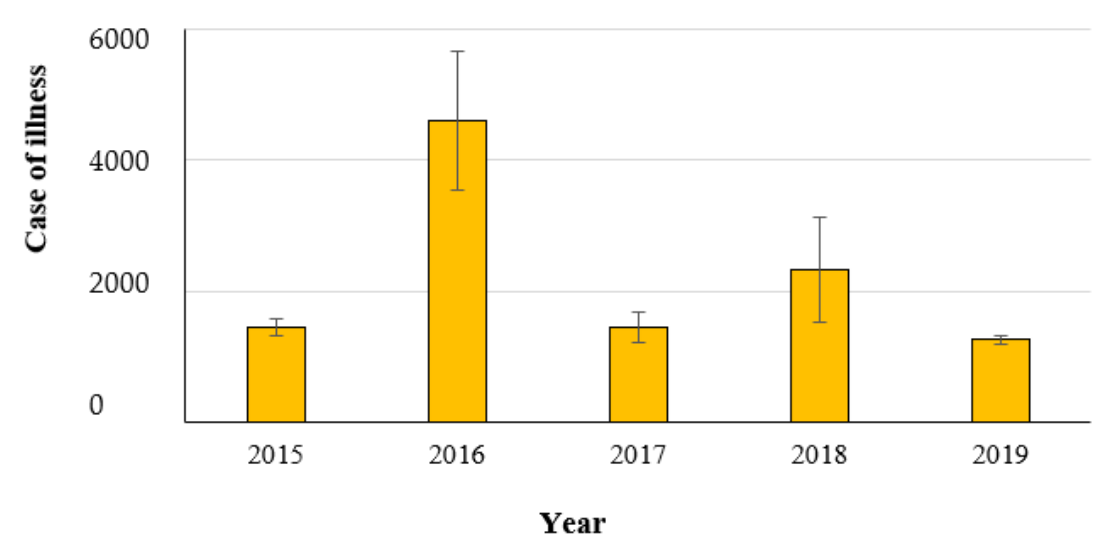

(a)

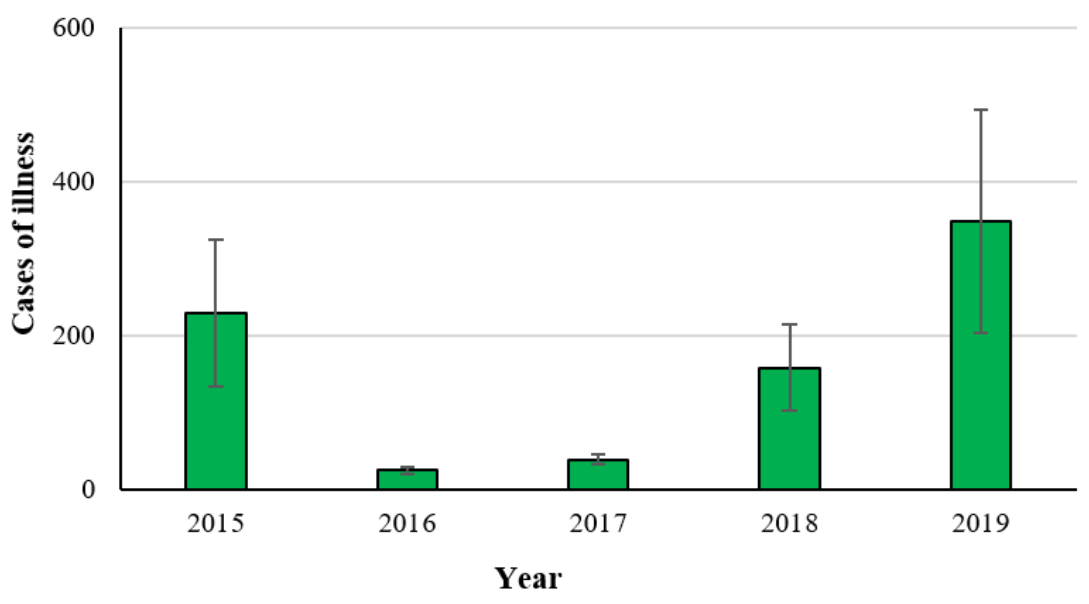

(c)

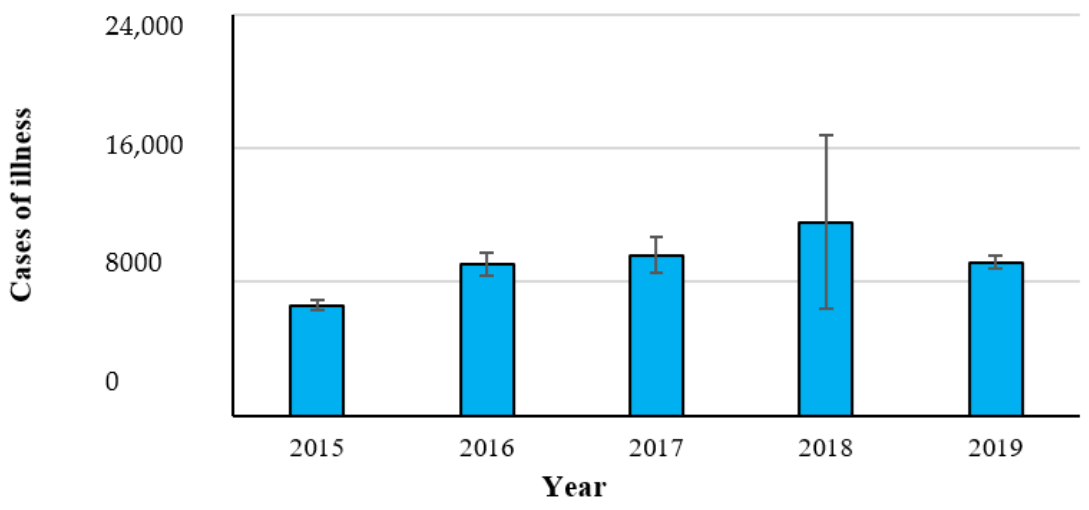

(b)

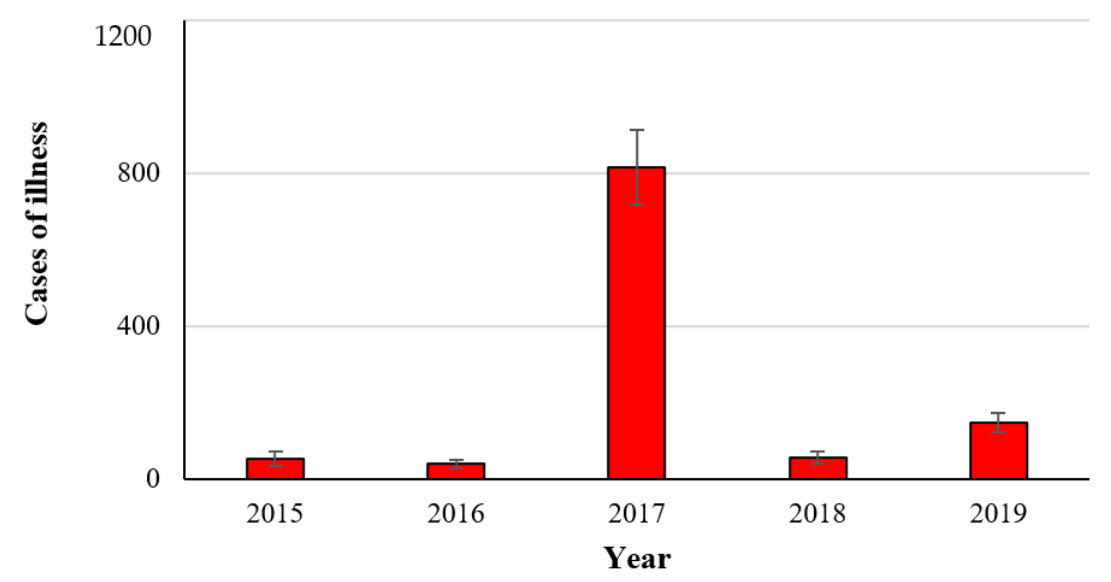

(d)

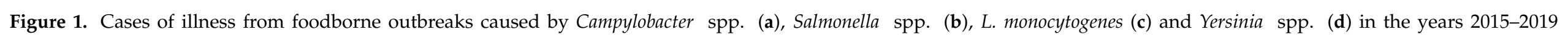
(EFSA-ECDC 2021). 


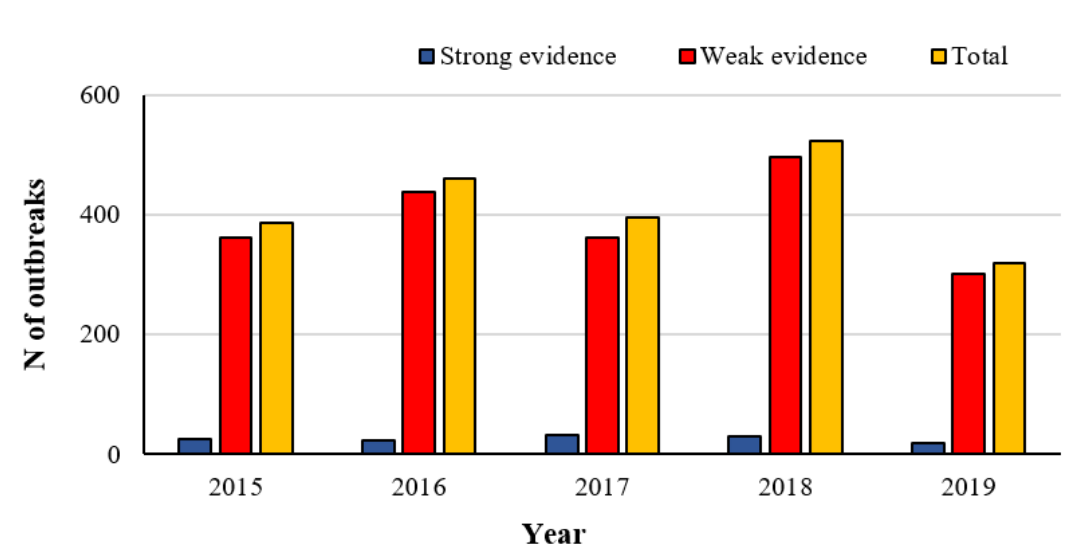

(a)

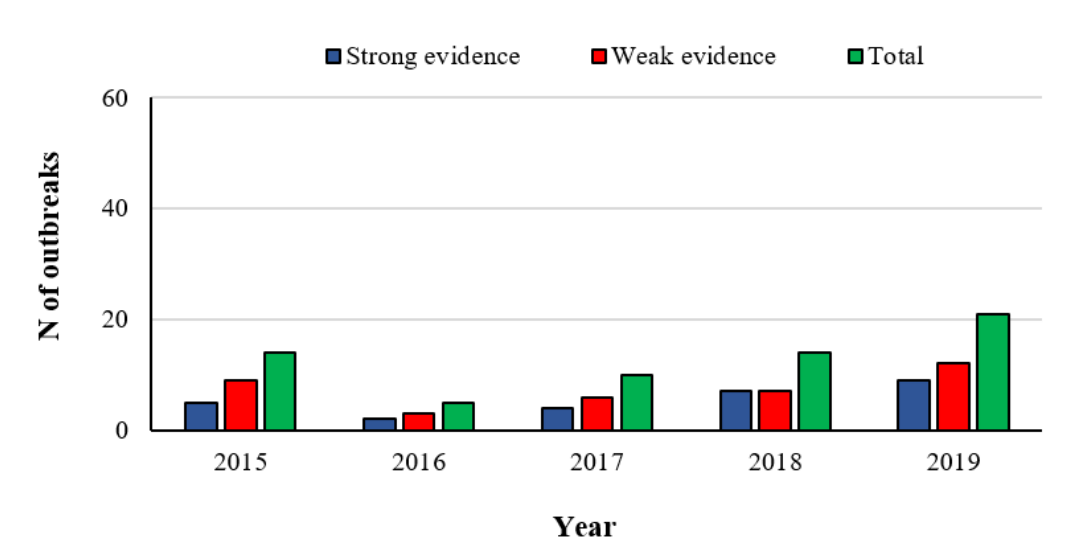

(c)

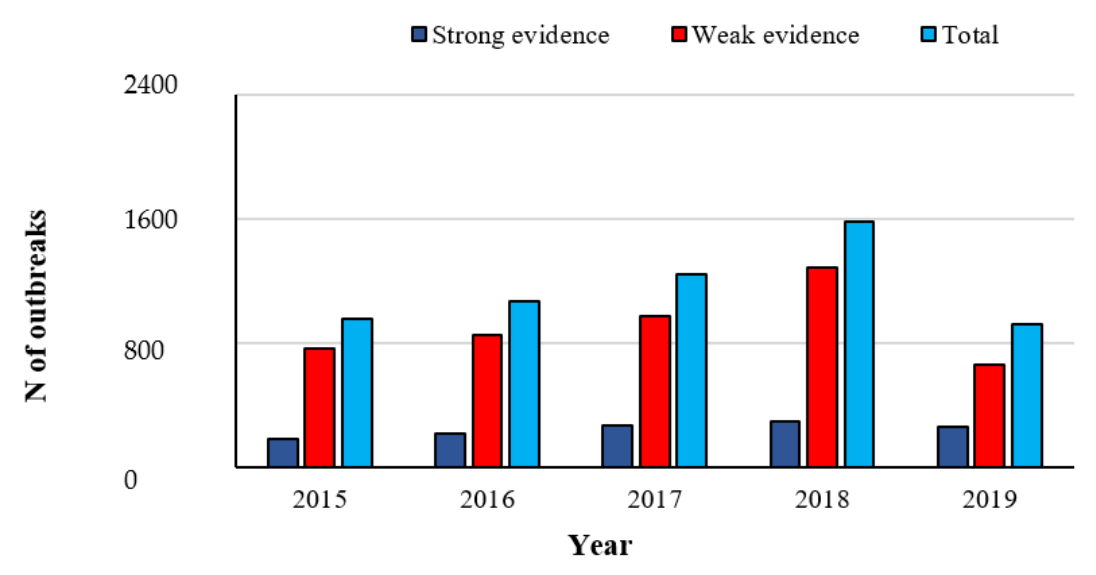

(b)

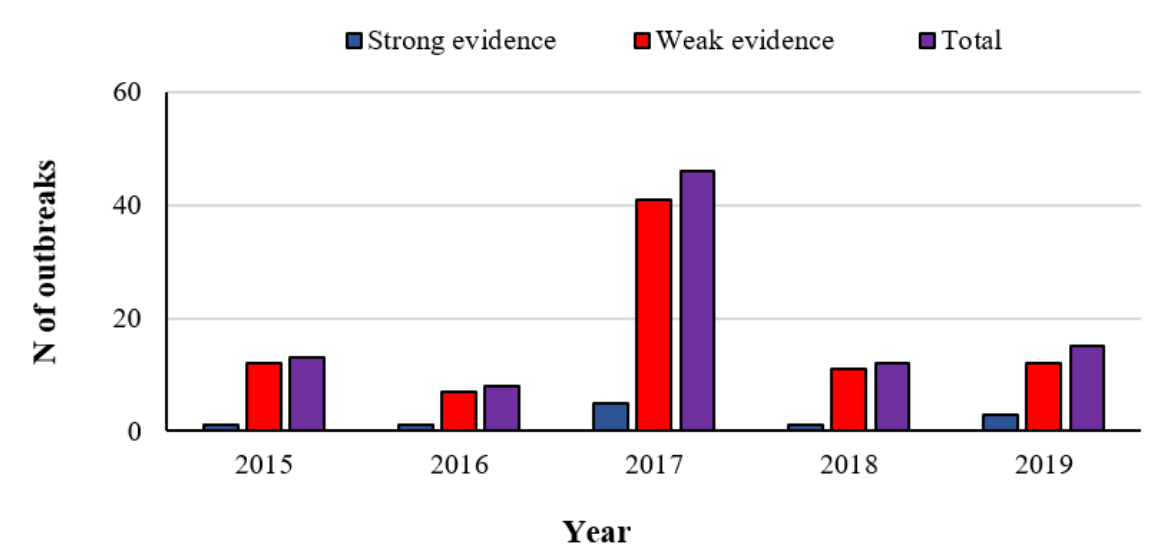

(d)

Figure 2. Number of foodborne outbreaks from Campylobacter spp. (a), Salmonella spp. (b), L. monocytogenes (c) and Yersinia spp. (d) in the years 2015-2019 (EFSA-ECDC 2021). 
Table 1. Overview of EU Member States signaling foodborne outbreaks in the years 2015-2019.

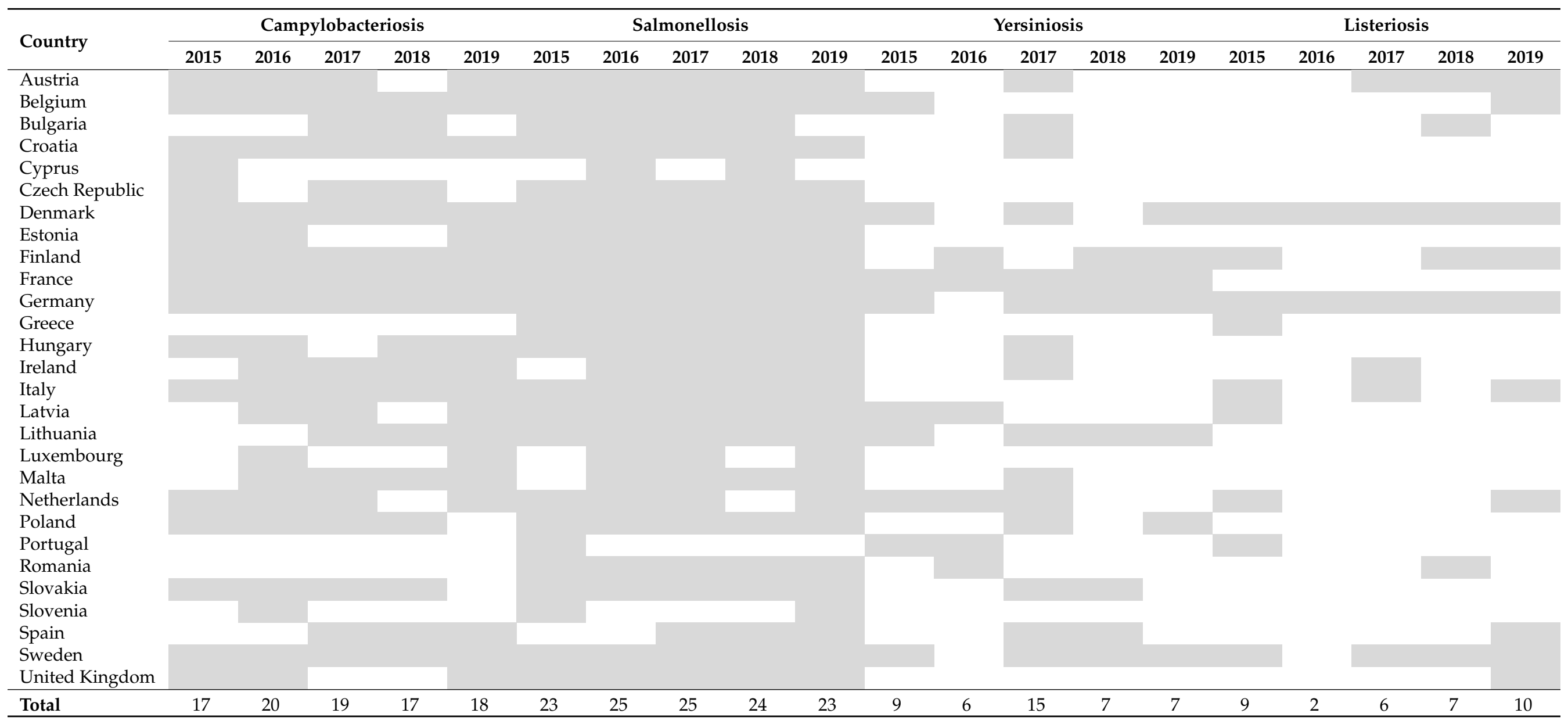




\subsection{Campylobacteriosis}

Campylobacteriosis is the most prevalent foodborne disease in many countries worldwide. The incidence increased in both developed and developing countries over the last 10 years, particularly in North America, Europe, and Australia, but it is becoming endemic also in some parts of Africa, Asia, and the Middle East. Some differences exist in the detection, as well as the standard and stringency of biocontrol protocols, surveillance bias, and availability of natural reservoirs [9]. Moreover, it seems to peak during the late spring and summer months, due to a combination of fluctuating infection rates in poultry and increased human exposure to environmental reservoirs as well as different eating and hygiene patterns during the summer months [10].

The illness is characterized by watery or bloody diarrhea, fever, vomiting, and stomach cramps, and other self-limiting gastrointestinal disorders [11]. However, various systemic infections, such as acute colitis and appendicitis, septic thrombophlebitis, endocarditis, neonatal sepsis, pneumonia, and bloodstream infections may also occur. Other major post-infections include severe demyelinating neuropathy, Guillain-Barré syndrome, Miller-Fisher syndrome, colorectal cancer, and Barrett's esophagus. In small group of patients, Campylobacter species can cause other extra-gastrointestinal infections such as brain abscesses, meningitis, lung infections, and reactive arthritis [12]. Campylobacter jejuni is the most frequently reported agent of campylobacteriosis, although other species such as Campylobacter coli, Campylobacter concisus, Campylobacter upsaliensis, Campylobacter ureolyticus, Campylobacter hyointestinalis and Campylobacter sputorum can be associated with such infection [11].

The number of confirmed human cases of campylobacteriosis revealed by the EFSAECDC Annual Reports in the years 2015-2019 is shown in Figure 3. It can be observed that the trend remained stable, and the decrease observed in the year 2019 compared to 2018 (59.7 versus 64.1 per 100,000 population) had no statistical significance $(p>0.05)$ by OneWay ANOVA (GraphPad InStat version 3.0). In 2019, 319 outbreaks involving 1254 cases of illness, 125 hospitalizations and no deaths were reported. The most common food sources for the strong-evidence outbreaks were broiler meat and milk, as in previous years [9]. In the years 2013-2016, a surveillance study performed in Italy revealed that C. jejuni was the most prevalent species causing gastroenteritis, and $45 \%$ of all the annual cases were reported in the summer period. Moreover, high rates of ciprofloxacin and tetracycline resistance in Campylobacter spp., as well as an increasing percentage of C. coli strains simultaneously resistant to ciprofloxacin, tetracycline and erythromycin were observed [13].

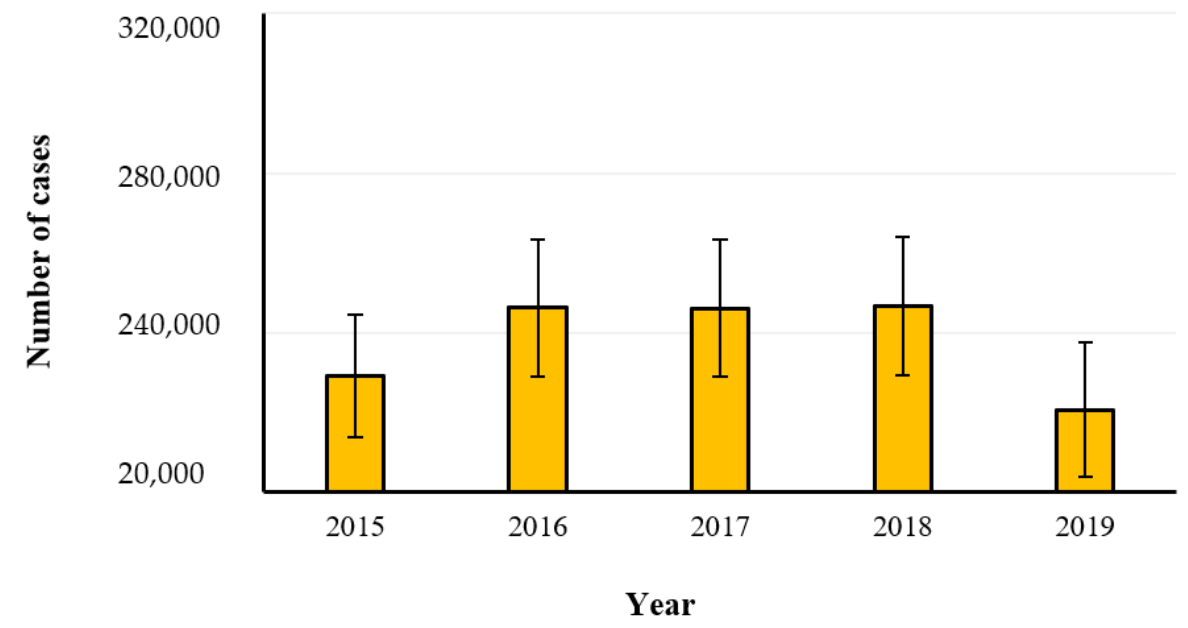

Figure 3. Number of confirmed human cases of campylobacteriosis in the years 2015-2019 (EFSA-ECDC 2021).

The main reservoir of Campylobacter spp. is the gastrointestinal tract of wild, domestic, farm, and pet animals [14] and it can survive under stress conditions by activating sev- 
eral survival mechanisms based on viable but non-culturable state, biofilm development, phase variation, genome reduction, and heterogeneity of the isolates. The consumption of contaminated poultry meat, milk products, as well as vegetables, seafood, water, or improperly cooked meat, is the main source of human campylobacteriosis [12]. The most important symptoms and food sources of campylobacteriosis are shown in Figure 4, while the food items associated with strong- and weak-evidence foodborne outbreaks occurred in the EU Member States in the year 2019 are reported in Figure 5.

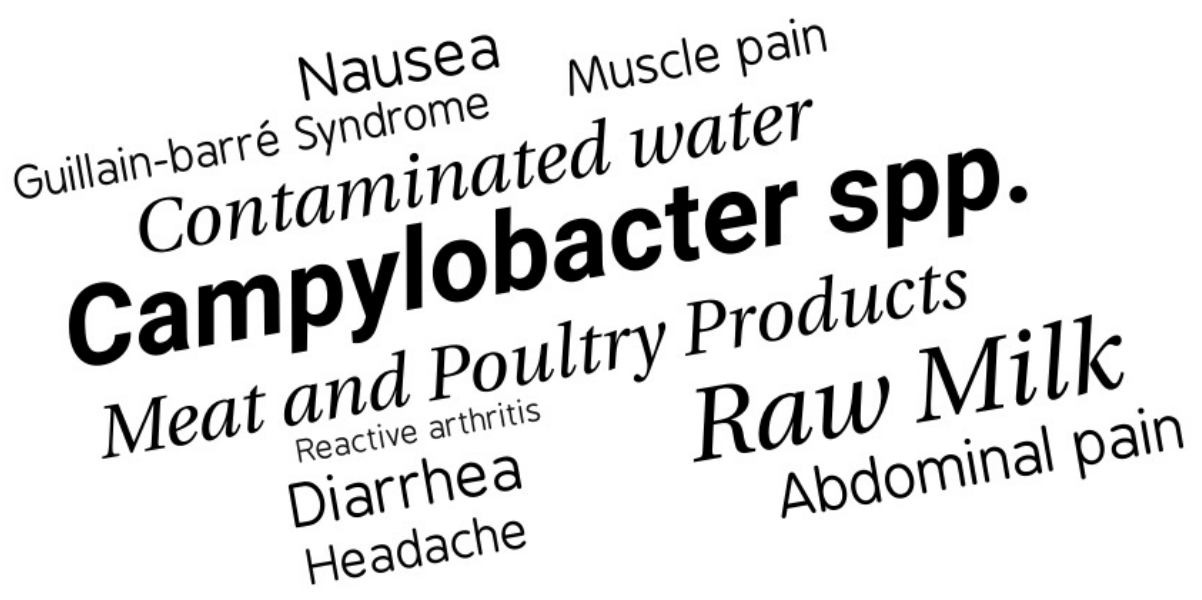

Figure 4. Symptomatology and food sources of Campylobacter spp. by a word cloud generator (www.wordart.com, accessed on 20 September 2021).

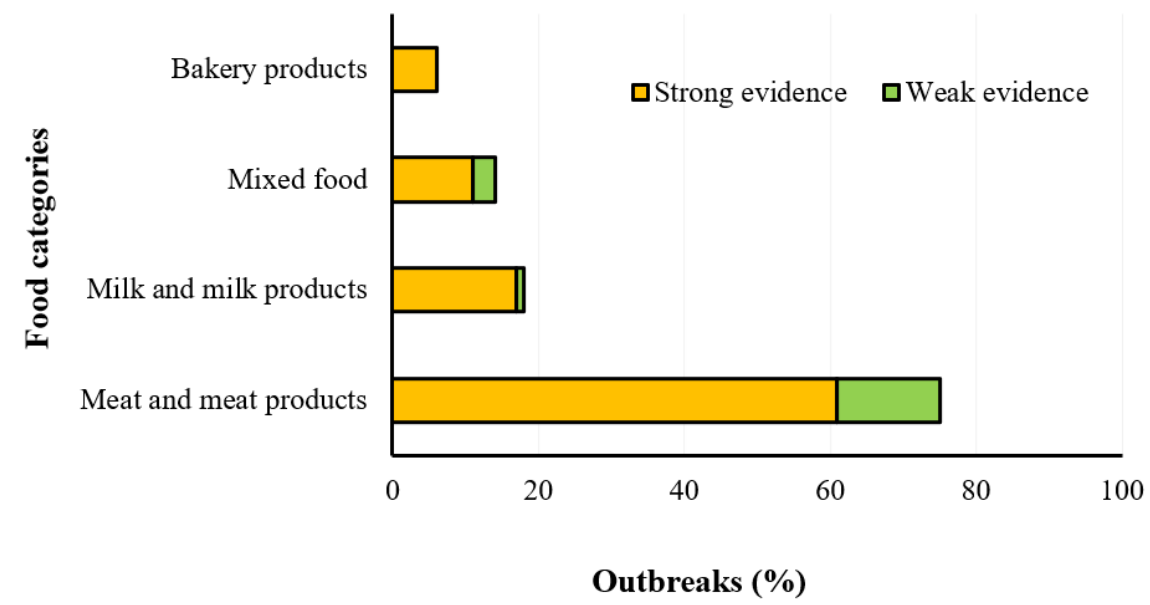

Figure 5. Distribution of different food items associated with strong- and weak-evidence foodborne outbreaks caused by Campylobacter spp. in the EU Member States in the year 2019 (EFSA-ECDC 2021).

\subsection{Salmonellosis}

Non-typhoidal Salmonella serotypes are among the major pathogens causing foodborne outbreaks worldwide. It has been reported that 93.8 million cases of non-typhoidal salmonellosis and 155,000 deaths occur every year in the world and that $86 \%$ of these illnesses are due to the consumption of Salmonella-contaminated foods. The main food items are eggs and egg products, chicken meat but also meat of other species, vegetables, and dairy products [15].

Two species of the genus Salmonella have been identified, Salmonella bongori and Salmonella enterica. The latter is distinguished in more than 2500 different serovars, and two of them, i.e., Enteritidis and Typhimurium are associated with most of Salmonella foodborne outbreaks worldwide [16]. Gastroenteritis is the most frequent clinical form of S. enterica infection and is characterized by nausea, headache, abdominal cramp, vomiting and fever, appearing after an incubation period of $8-48 \mathrm{~h}$. The diarrheal stools are generally 
non-bloody and of moderate volume, even if large volumes of watery stools and dysentery may occur. The stool culture can give a positive result for $4-5$ weeks after the infection, and for $>1$ year in chronic carriers [17]. The symptoms and food sources for Salmonella spp. infection are shown in Figure 6.

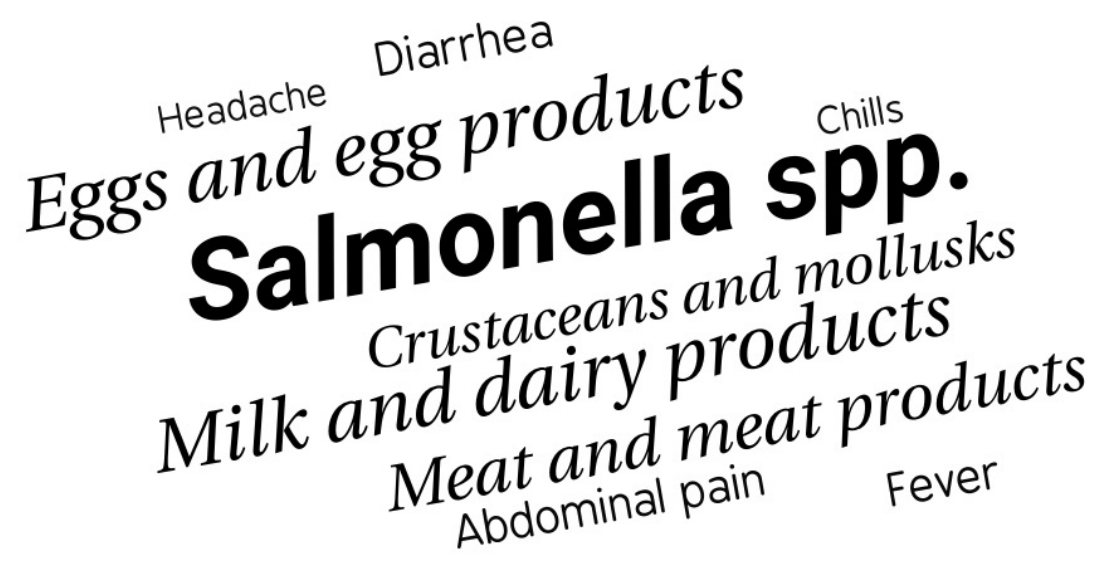

Figure 6. Symptomatology and food sources of Salmonella spp. by a word cloud generator (www. wordart.com, accessed on 20 September 2021).

Salmonellosis is the second most common gastrointestinal infection in humans after campylobacteriosis, and 87,923 confirmed cases were reported in the year 2019, with a notification rate of 20.0 cases per 100,000 population. The hospitalization cases corresponded to 1,915 in the year 2019 and ranged from 1719 to 2298 in the remaining investigated years. The trend was stable over the last 5 years (Figure 7) after a long period of declining [8]. The statistical analysis by One-Way ANOVA (GraphPad InStat version 3.0) showed no significance $(p>0.05)$ among the years. The most involved food sources are eggs and egg products, meat and meat products, especially poultry and pork meat, but also bakery products, and other mixed food (Figure 8). Salmonella enterica is a low-infectious dose microorganism that can grow even in low-water activity foods, and cases of foodborne diseases have been recently associated with infant formula and dried milk products, almonds, peanut butter, chocolate, and spices [18]. The microorganism has been found also in the plant environment, crops, bean sprouts, coriander, spinach, ground cinnamon, garlic powder, onion powder, red chili pepper powder and green tea. The aquatic environment is also an important source of salmonellosis by consumption of fresh fish, oysters, shrimps, and frog legs, especially in areas contaminated with fecal matter [16].

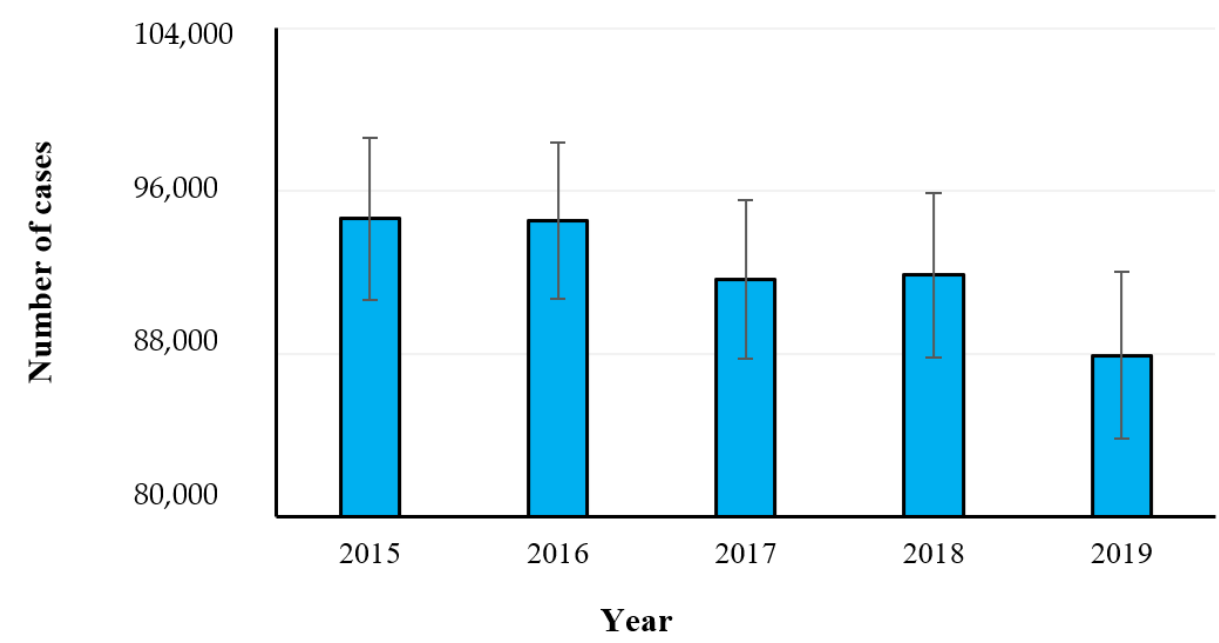

Figure 7. Number of confirmed human cases of salmonellosis in the years 2015-2019 (EFSAECDC 2021). 


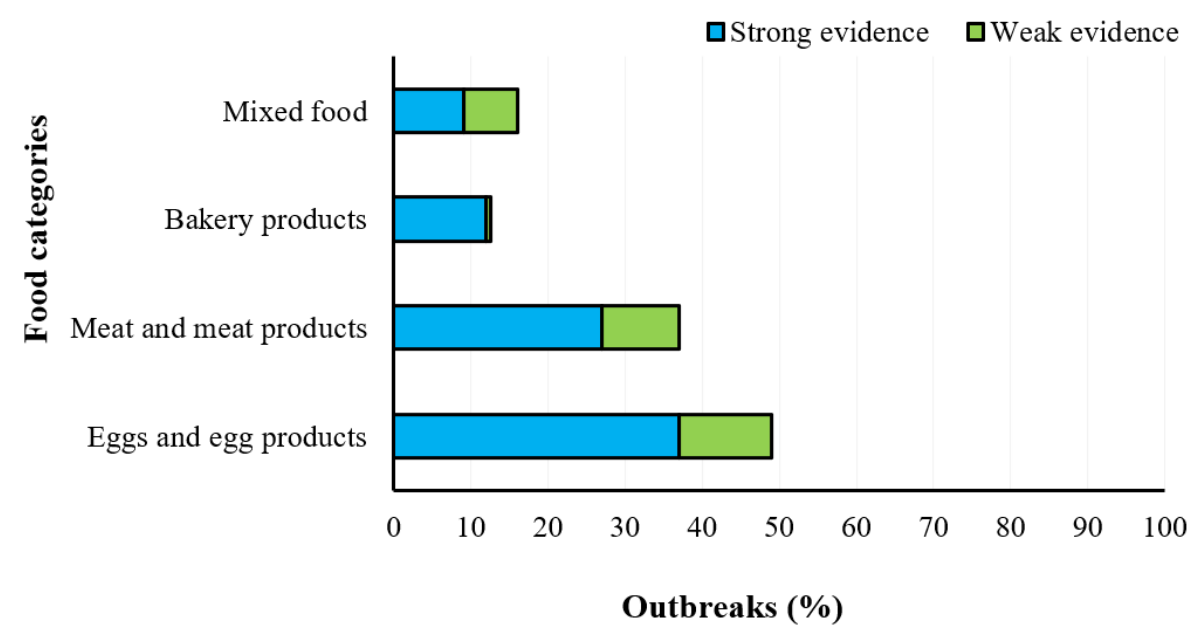

Figure 8. Distribution of different food items associated with strong- and weak-evidence foodborne outbreaks caused by Salmonella spp. in the EU Member States in the year 2019 (EFSA-ECDC 2021).

\subsection{Listeriosis}

Listeria monocytogenes is an environmentally ubiquitous foodborne pathogen with a high mortality rate (20-30\%). It can survive in the acidic environment of the stomach as well as bile salts, colonize and invade the host epithelium, spreading from infected cells to neighboring cells and then to other organs, and leading to severe complications, such as meningitis, septicemia, and maternal-fetal infections. Listeriosis is particularly life-threatening in high-risk groups, i.e., immunocompromised individuals and pregnant women [19]. In the year 2019, 2621 confirmed invasive human cases with an EU notification rate of 0.46 cases per 100,000 population were reported, while the hospitalization cases were 236. The trend remained stable in the years 2015-2019 after a long period of an increasing trend (Figure 9). The One-Way ANOVA (GraphPad InStat version 3.0) performed among the investigated years showed no statistical significance $(p>0.05)$. The overall case fatality was high (17.6\%) and increased compared with 2018 and 2017 (13.6 and 15.6\%, respectively). This makes listeriosis one of the most serious foodborne diseases under EU surveillance [8]. The reported incidence of such disease in industrialized countries has increased dramatically since the 1980s and tracing-back with forward investigation are particularly recommended. The Program for Monitoring Emerging Diseases described 123 events from 1996 through 2018, of which 81 (65\%) were associated with two or more human cases (outbreak events), 13 (11\%) were linked to only one human case (sporadic cases), and $29(24 \%)$ were precautionary food product recalls without associated human cases. The implicated food vehicle was identified in 69 (85\%) outbreak events and in $10(77 \%)$ sporadic case events [20]. The most commonly foods connected with listeriosis are ready-to-eat products being high in protein, with moderate water activity and low background microflora. Moreover, the presence and persistence of such pathogen in the food production environments is a particular concern, leading to contamination of multiple batches of products over a long period of time, as it can remain undetected in harborage sites, commonly known as niches. Such sites are often difficult to reach and clean with normal sanitation procedures, providing growth favorable conditions where L. monocytogenes can colonize. Furthermore, it can form biofilms on food production surfaces that are particularly resistant to disinfection [21]. In Figure 10 the symptomatology and food sources of listeriosis are shown, while the food items associated with foodborne outbreaks reported in the year 2019 are described in Figure 11. 


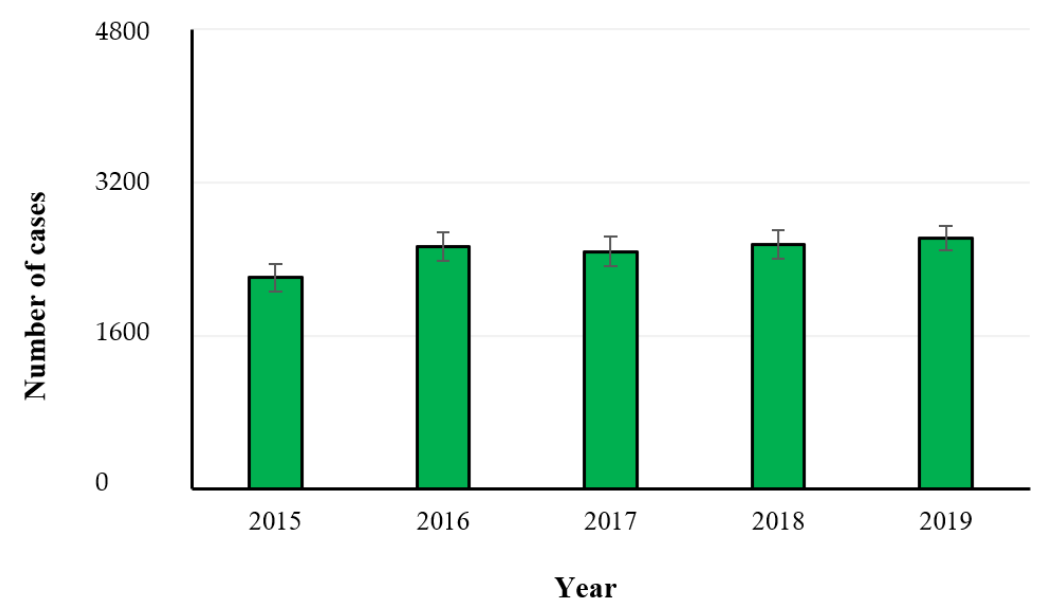

Figure 9. Number of confirmed human cases of listeriosis in the years 2015-2019 (EFSA-ECDC 2021).

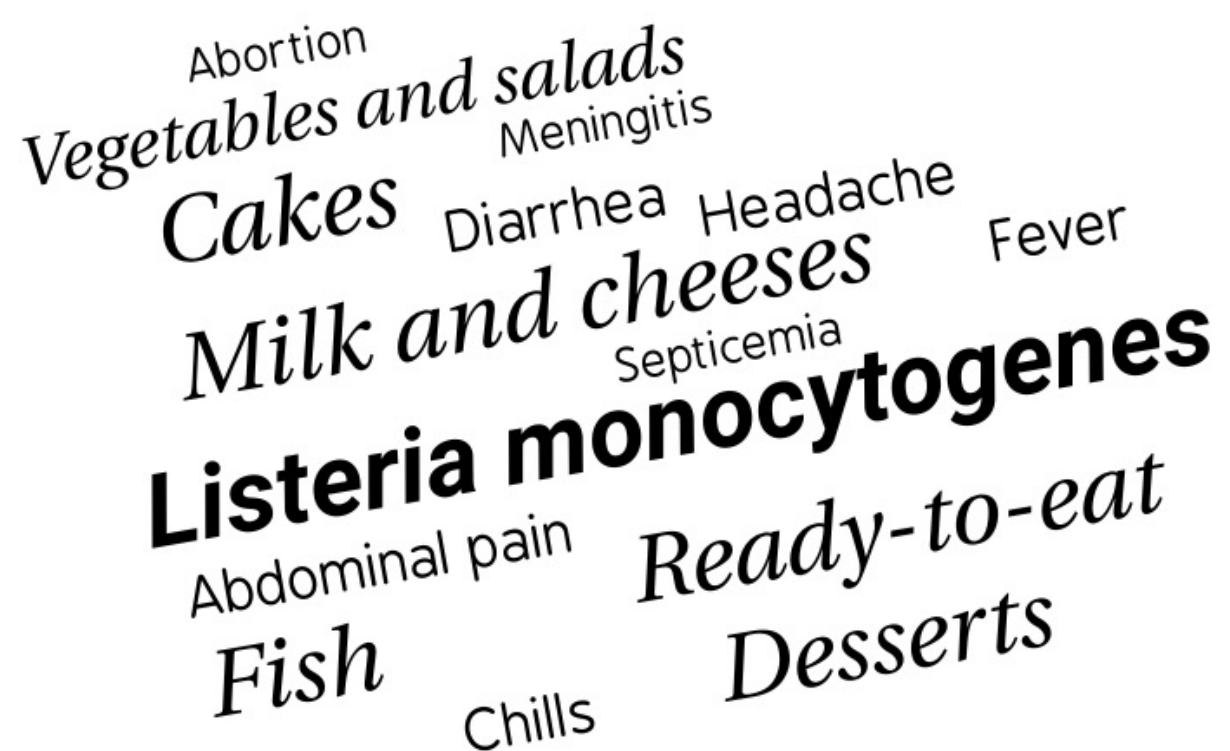

Figure 10. Symptomatology and food sources of L. monocytogenes by a word cloud generator (www.wordart.com, accessed on 20 September 2021).

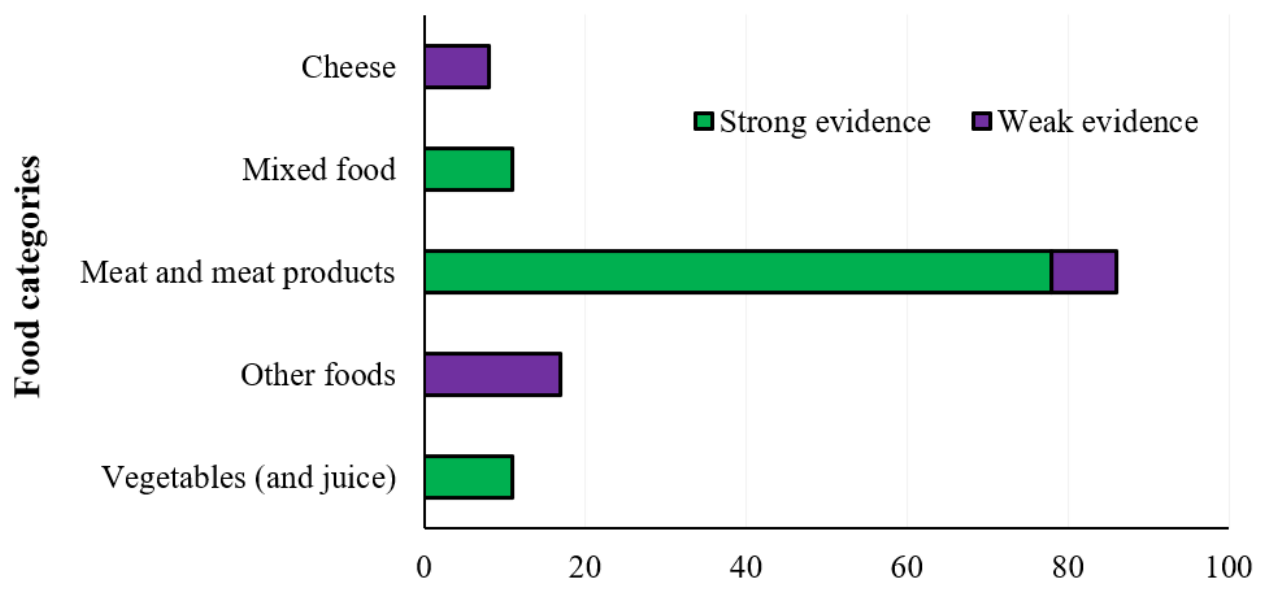

Outbreaks (\%)

Figure 11. Distribution of different items associated with strong- and weak-evidence foodborne outbreaks caused by L. monocytogenes in the EU Member States in the year 2019 (EFSA-ECDC 2021). 


\subsection{Yersiniosis}

There are 21 species belonging to the genus Yersinia, and three of them are currently considered human pathogenic microorganisms, i.e., Yersinia pestis, Yersinia pseudotuberculosis and Yersinia enterocolitica. The latter is the main responsible of the foodborne disease yersiniosis, that is characterized by diarrhea, abdominal pain, and fever. Other uncommon symptoms, such as reactive arthritis and nodular erythema, are also described. The US Department of Health and Human Services/Centers for Disease Control and Prevention showed a significant increase of $166 \%$ in the incidence of Yersinia spp. circulation in 2017 compared to that in the period between 2014 and 2016 [22]. In the year 2019, outbreaks and illnesses caused by Yersinia spp. (15 and 149, respectively) were reported by seven EU Member States (i.e., Denmark, Finland, France, Germany, Lithuania, Poland, and Sweden) and Y. enterocolitica was identified as the causative agent in all these outbreaks. The trend of yersiniosis occurring in the EU Member States in the years 2015-2019 is presented in Figure 12. The statistical analysis of data could not be made as the values reported for some years did not refer only to such microorganism. In the year 2017, Yersinia spp. was reported with other bacterial agents comprising Aeromonas hydrophila, Enteroaggregative E. coli, Enterotoxigenic E. coli, Enteroinvasive E. coli, Enteropathogenic E. coli, Shigella flexneri, Shigella sonnei and other unspecified bacteria. The most represented food categories (Figure 13) responsible of strong-evidence foodborne outbreaks were pig meat and products thereof, and vegetables and juices and other products thereof [8]. With regards to the microorganism, the Eastern Mediterranean regions as well as Africa has the first and second rank of prevalence, while Europe is characterized by the least prevalence in gastroenteritis cases. Yersiniosis in also reported in US, Brazil, and Australia [23].

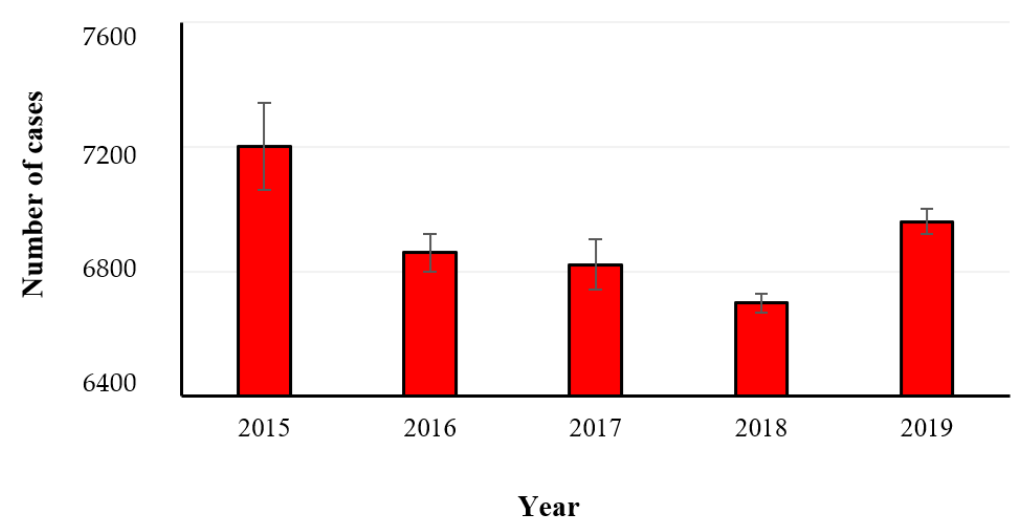

Figure 12. Number of confirmed human cases of yersiniosis in the years 2015-2019 (EFSA-ECDC 2021).

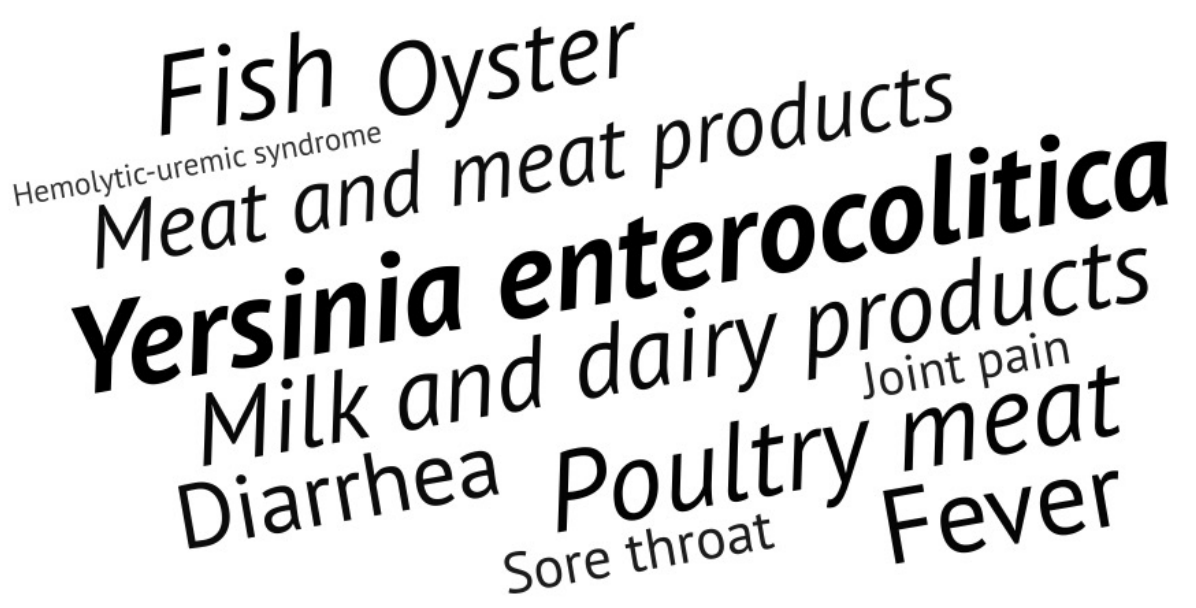

Figure 13. Symptomatology and food sources of $Y$. enterocolitica by a word cloud generator (www.wordart.com, accessed on 20 September 2021). 


\section{Prevention and Control Strategies}

The prevention of food contamination with foodborne pathogens represents the most important tool to overcome foodborne diseases. Different measures can be applied at farming or planting level for foods of animal and vegetal origin, respectively. In the first case, the use of probiotics and/or antibiotics, vaccination, and other procedures aiming at protecting the animal health are the best strategies to be performed. Instead, for fresh produce the implementation of good agricultural practices before planting, during production, and after harvest are particularly expected. Moreover, the application of a system that states the responsibility for food safety on each stage involved in the food supply chain and aiming at preventing/eliminating food safety hazards or reducing them to an acceptable level is mandatory [2]. Several countries have implemented microbiological criteria (MC) to reduce the foodborne diseases. According to the Regulation (EC) No 2073/2005 and following amendments, the EU established MC for many pathogens and food products, while in the US, Salmonella performance standards for selected meat and poultry products were set as part of the Pathogen Reduction/Hazard Analysis and Critical Control Point (HACCP) Systems Final Rule [24]. Recently, New Zealand fixed MC for Campylobacter spp. contamination in poultry products, and consequently some reductions in human illnesses were observed [25].

The World Trade Organization and Codex Alimentarius proposed some strategies to safeguard consumers by the application of the Appropriate Level of Protection (ALOP, also known as ALR, Acceptable Level of Risk) and the Food Safety Objective (FSO). The first corresponds to the level of protection that is considered acceptable to protect human, animal or plant health. Instead, the Tolerable Level of Risk (TLR) is the risk that can be tolerated by society, in comparison with the other significant risks of daily life. Both ALR and TLR can be considered reasonable estimates based on outbreak investigations and surveillance studies. Instead, the FSO can be proposed for any food hazard to reduce the level of risk in the production and distribution environments. Moreover, the food companies and distribution chains specifically apply the HACCP system, as well as other good practices, to control all significant hazards associated with a specific food (Figure 14) and these strategies represent practical control measures to reduce the level of risk in the food production sector [26].

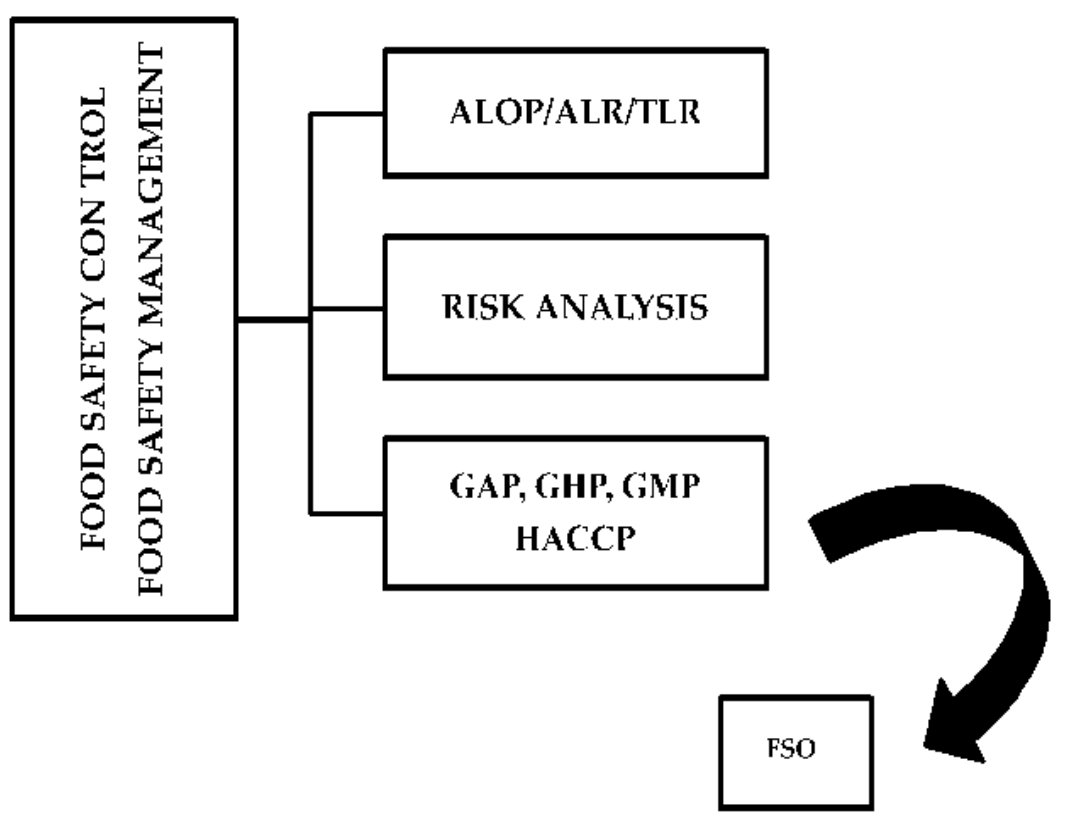

Figure 14. Food safety management and control strategies. ALOP = Appropriate Level of Protection; ALR = Acceptable Level of Risk; TLR = Tolerable Level of Risk; GAP = Good Agricultural Practices; GHP = Good Hygiene Practices; GMP = Good Manufacturing Practices; FSO = Food Safety Objective . 
According to the Regulation (EC) No 852/2004 on the hygiene of foodstuffs, the primary responsibility for food safety rests with the food business operators that shall establish and apply food safety procedures based on the HACCP principles. Such a preventive approach has been adopted in the US too, with the publication of the FDA Food Safety Modernization Act in 2011. Among such preventive measures, the monitoring of the food industry environmental microbiome can represent an important tool to improve the quality assessment in the food production chain [27]. By contrast, the traditional physical treatments of foods, that may efficiently deactivate the foodborne pathogens, can affect their functional and sensory characteristics, while the use of chemical agents such as antibiotics can contribute to the large spread of antibiotic resistance, by transferring resistant genes from antibiotic-resistant microorganisms to the human body [28].

In conclusion, the generation of new structured strategies in the food safety assessment can greatly assist the food industry to develop improved sustainable production chains and prevent foodborne outbreak incidents.

Author Contributions: Conceptualization, M.S. and P.V.; investigation, M.S.; writing-original draft preparation, P.V.; writing-review and editing, M.S. and P.V. All authors have read and agreed to the published version of the manuscript.

Funding: This research received no external funding.

Conflicts of Interest: The authors declare no conflict of interest.

\section{References}

1. AL-Mamun, M.; Chowdhury, T.; Biswas, B.; Absar, N. Food Poisoning and Intoxication: A Global Leading Concern for Human Health. In Food Safety and Preservation. Modern Biological Approaches to Improving Consumer Health; Grumezescu, A.M., Holban, A.M., Eds.; Elsevier Inc.: Amsterdam, The Netherlands, 2018; Chapter 11; pp. 307-352. [CrossRef]

2. Chaves, R.D.; Alvarenga, V.O.; Campagnollo, F.B.; Rodriguez Caturla, M.Y.; Oteiza, J.M.; Sant'Ana, A.S. Food Safety. In Current Developments in Biotechnology and Bioengineering-Food and Beverages Industry; Part 1: Food and, Fermentation; Pandey, A., Sanroman, R.A., Du, G., Soccol, C.R., Dussap, C.-G., Eds.; Elsevier Inc.: Amsterdam, The Netherlands, 2017; Chapter 9; pp. 245-259.

3. Mota, J.D.O.; Boué, G.; Prévost, H.; Maillet, A.; Jaffres, E.; Maignien, T.; Arnich, N.; Sanaa, M.; Federighi, M. Environmental monitoring program to support food microbiological safety and quality in food industries: A scoping review of the research and guidelines. Food Control. 2021, 130, 108283. [CrossRef]

4. Yu, H.; Guo, W.; Lu, X.; Xu, H.; Yang, Q.; Tan, J.; Zhang, W. Reduced graphene oxide nanocomposite based electrochemical biosensors for monitoring foodborne pathogenic bacteria: A review. Food Control. 2021, 127, 108117. [CrossRef]

5. Shan, L.; Wang, S.; Wu, L.; Tsai, F.-S. Cognitive Biases of Consumers' Risk Perception of Foodborne Diseases in China: Examining Anchoring Effect. Int. J. Environ. Res. Public Health 2019, 16, 2268. [CrossRef] [PubMed]

6. Ebel, E.D.; Williams, M.S.; Ward-Gokhale, L.A.; Kisselburgh, H.M. Assessing the maximum size of annual foodborne outbreaks in the United States: An analysis of 1973-2016 outbreaks. Microb. Risk Anal. 2019, 12, 20-26. [CrossRef]

7. EFSA (European Food Safety Authority). Update of the technical specifications for harmonised reporting of food-borne outbreaks through the European Union reporting system in accordance with Directive 2003/99/EC. EFSA J. 2014, 12, 3598.

8. EFSA-ECDC (European Food Safety Authority and European Centre for Disease Prevention and Control). The European Union One Health 2019 Zoonoses Report. EFSA J. 2021, 19, 6406.

9. Kaakoush, N.O.; Castaño-Rodríguez, N.; Mitchell, H.M.; Man, S.M. Global Epidemiology of Campylobacter Infection. Clin. Microbiol. Rev. 2015, 28, 687-720. [CrossRef] [PubMed]

10. Kuhn, K.G.; Nygård, K.M.; Guzman-Herrador, B.; Sunde, L.S.; Rimhanen-Finne, R.; Trönnberg, L.; Jepsen, M.R.; Ruuhela, R.; Wong, W.K.; Ethelberg, S. Campylobacter infections expected to increase due to climate change in Northern Europe. Sci. Rep. 2020, 10, 13874. [CrossRef]

11. Sher, A.A.; Ashraf, M.A.; Mustafa, B.E.; Raza, M.M. Epidemiological trends of foodborne Campylobacter outbreaks in the United States of America, 1998-2016. Food Microbiol. 2021, 97, 103751. [CrossRef]

12. Igwaran, A.; Okoh, A. Human campylobacteriosis: A public health concern of global importance. Heliyon 2019, 5, e02814. [CrossRef] [PubMed]

13. García-Fernández, A.; Dionisi, A.M.; Arena, S.; Iglesias-Torrens, Y.; Carattoli, A.; Luzzi, I. Human Campylobacteriosis in Italy: Emergence of Multi-Drug Resistance to Ciprofloxacin, Tetracycline, and Erythromycin. Front. Microbiol. 2018, 9, 1906. [CrossRef]

14. Endtz, H.P. Campylobacter infections. In Hunter's Tropical Medicine and Emerging Infectious Disease, 10th ed.; Philadelphia, P.A., Ryan, E.T., Hill, D.R., Solomon, T., Aaronson, N.E., Endy, T.P., Eds.; Elsevier Inc.: Amsterdam, The Netherlands, 2020; Chapter 50; pp. 507-511. [CrossRef] 
15. Sher, A.A.; Mustafa, B.E.; Grady, S.C.; Gardiner, J.C.; Saeed, A.M. Outbreaks of foodborne Salmonella enteritidis in the United States between 1990 and 2015: An analysis of epidemiological and spatial-temporal trends. Int. J. Infect. Dis. 2021, 105 , 54-61. [CrossRef] [PubMed]

16. Agregán, R.; Munekata, P.E.; Zhang, W.; Zhang, J.; Pérez-Santaescolástica, C.; Lorenzo, J.M. High-pressure processing in inactivation of Salmonella spp. in food products. Trends Food Sci. Technol. 2021, 107, 31-37. [CrossRef]

17. Nadi, Z.R.; Salehi, T.Z.; Tamai, I.A.; Foroushani, A.R.; Sillanpaa, M.; Dallal, M.M.S. Evaluation of antibiotic resistance and prevalence of common Salmonella enterica serovars isolated from foodborne outbreaks. Microchem. J. 2020, 155, 104660. [CrossRef]

18. Dhaliwal, H.K.; Gänzle, M.; Roopesh, M. Influence of drying conditions, food composition, and water activity on the thermal resistance of Salmonella enterica. Food Res. Int. 2021, 147, 110548. [CrossRef] [PubMed]

19. Aljasir, S.F.; D'Amico, D.J. Effect of pre-exposure to protective bacterial cultures in food on Listeria monocytogenes virulence. LWT Food Sci. Technol. 2021, 152, 112373. [CrossRef]

20. Desai, A.N.; Anyoha, A.; Madoff, L.C.; Lassmann, B. Changing epidemiology of Listeria monocytogenes outbreaks, sporadic cases, and recalls globally: A review of ProMED reports from 1996 to 2018. Int. J. Infect. Dis. 2019, 84, 48-53. [CrossRef]

21. Evans, E.; Samuel, E.; Redmond, E.; Taylor, H. Exploring Listeria monocytogenes perceptions in small and medium sized food manufacturers: Technical leaders' perceptions of risk, control and responsibility. Food Control. 2021, 126, 108078. [CrossRef]

22. Wang, J.; Liu, M.; Wang, H.; Wu, Q.; Ding, Y.; Xu, T.; Ma, G.; Zhong, Y.; Zhang, J.; Chen, M.; et al. Occurrence, molecular characterization, and antimicrobial susceptibility of Yersinia enterocolitica isolated from retail food samples in China. LWT Food Sci. Technol. 2021, 150, 111876. [CrossRef]

23. Riahi, S.M.; Ahmadi, E.; Zeinali, T. Global prevalence of Yersinia enterocolitica in cases of gastroenteritis: A systematic review and meta-analysis. Int. J. Microbiol. 2021, 2021, 1499869. [CrossRef]

24. Lambertini, E.; Ruzante, J.M.; Chew, R.; Apodaca, V.L.; Kowalcyk, B.B. The public health impact of different microbiological criteria approaches for Salmonella in chicken parts. Microb. Risk Anal. 2019, 12, 44-59. [CrossRef]

25. New Zealand Ministry for Primary Industries. Campylobacter Risk Management Strategy 2017-2020; Ministry for Primary Industries: Wellington, New Zealand, 2017.

26. Paparella, A. Food safety: Definitions and aspects. In Food Safety Hazards; Al-Rub, F.A., Shibhab, P., Al-Rub, S.A., Pittia, P., Paparella, A., Eds.; Gavin Ebooks: Lisle, IL, USA, 2020; pp. 1-5. [CrossRef]

27. De Filippis, F.; Valentino, V.; Alvarez-Ordóñez, A.; Cotter, P.D.; Ercolini, D. Environmental microbiome mapping as a strategy to improve quality and safety in the food industry. Curr. Opin. Food Sci. 2021, 38, 168-176. [CrossRef]

28. Hou, J.; Luo, R.; Ni, H.; Li, K.; Mgomi, F.C.; Fan, L.; Yuan, L. Antimicrobial potential of kombucha against foodborne pathogens: A review. Qual. Assur. Saf. Crop. Foods 2021, 13, 53-61. [CrossRef] 